\title{
Angular Structure of Lacunarity, and the Renormalization Group
}

\author{
R. C. Ball, ${ }^{1}$ G. Caldarelli, ${ }^{2,3}$ and A. Flammini ${ }^{1,2}$ \\ ${ }^{1}$ Department of Physics, University of Warwick, CV4 7AL Coventry, United Kingdom \\ ${ }^{2}$ TCM Group, Cavendish Laboratory, University of Cambridge, CB3 OHE Cambridge, United Kingdom \\ ${ }^{3}$ INFM-Università di Roma 1 "La Sapienza," Piazzale Aldo Moro 2, 00185 Roma, Italy
}

(Received 24 March 2000)

\begin{abstract}
We formulate the angular structure of lacunarity in fractals, in terms of a symmetry reduction of the three point correlation function. This provides a rich probe of universality, and first measurements yield new evidence in support of the equivalence between self-avoiding walks (SAW's) and percolation perimeters in two dimensions. We argue that the lacunarity reveals much of the renormalization group in real space. This is supported by exact calculations for random walks and measured data for percolation clusters and SAW's. Relationships follow between exponents governing inward and outward propagating perturbations, and we also find a very general test for the contribution of long-range interactions.
\end{abstract}

PACS numbers: 64.60.Ak, 05.40.Fb

In this Letter we bring together two outstanding issues in the theory of fractals, which we believe will have bearing on critical phenomena more generally. The first is "lacunarity" [1-3], which as originally introduced could entail all the discriminations of fractal structure (beyond dimension) which are evidently visible yet hard to codify in a simple generic way. The second is the renormalization group (RG) [4] which has been a predominant influence for three decades in the theory of critical scaling phenomena, fractals included, yet has never to our knowledge been regarded as a directly measurable object in its own right. Here we present evidence and arguments that a particular version of lacunarity measurement is effectively a measurement of the (linearized) renormalization group.

The "lacunarity function" was introduced [5] as a scaling reduction of the three point correlation function for a statistical fractal,

$$
\mathcal{L}(R, \theta)=\frac{C_{3}\left(\vec{r}_{1}, \vec{r}_{2}, \vec{r}_{3}\right)}{C_{2}\left(\vec{r}_{1}, \vec{r}_{2}\right) C_{2}\left(\vec{r}_{1}, \vec{r}_{3}\right)},
$$

where $C_{3}\left(\vec{r}_{1}, \vec{r}_{2}, \vec{r}_{3}\right)$ is the conditional probability for points $\vec{r}_{2}$ and $\vec{r}_{3}$ to be occupied given that $\vec{r}_{1}$ is occupied, $C_{2}\left(\vec{r}_{1}, \vec{r}_{2}\right)$ is the conditional probability for point $\vec{r}_{2}$ occupied given $\vec{r}_{1}$ is occupied, and the lacunarity function $\mathcal{L}$ depends only on the angle $\theta$ between the two vectors $\vec{r}_{2}-\vec{r}_{1}$ and $\vec{r}_{3}-\vec{r}_{1}$ and their length ratio $R=r_{13} / r_{12}$.

The lacunarity function defined above is (by construction) independent of absolute length scale and is a pure critical-point scaling object. It is crucial that we are discussing measurements averaged over an ensemble of fractal objects, or at least self-averaging over the interior of one object much larger than both $r_{12}$ and $r_{13}$. The dependence of $\mathcal{L}$ on only a ratio of lengths follows from the assumption of continuous scale invariance, while the dependence on only a single internal angle follows in the more restrictive assumption that the ensemble of fractals in question has no favored directions. The whole analysis presupposes definition of some suitable measure for the fractal structure in question, but provided this is not multifractal [6,7] any simple ambiguity in the measure is expected to cancel from the lacunarity function.

I. The angular structure of lacunarity.-We introduce here the decomposition of the lacunarity function over angular harmonics, for example, in two dimensions

$$
\mathcal{L}(R, \theta)=\mathcal{L}_{0}(R)+2 \sum_{m=1}^{\infty} \mathcal{L}_{m}(R) \cos m \theta .
$$

Our physical interpretation of the individual components $\mathcal{L}_{m}\left(r^{\prime} / r\right)$ is that they measure the correlation between different length scales $r$ and $r^{\prime}$ of relative fluctuations, of a particular angular harmonic, in the local two point correlation about each mass point. They can be more precisely calculated as ratios

$$
\mathcal{L}_{m}\left(r^{\prime} / r\right)=M_{m}\left(r^{\prime}, r\right)=\frac{\left\langle S_{m}\left(r^{\prime} ; \vec{r}_{1}\right) S_{m}\left(r ; \vec{r}_{1}\right)^{*}\right\rangle}{\left\langle S_{0}\left(r^{\prime} ; \vec{r}_{1}\right)\right\rangle\left\langle S_{0}\left(r ; \vec{r}_{1}\right)\right\rangle},
$$

where $S_{m}\left(r ; \vec{r}_{1}\right)$ is the weighted count of occupied points within a shell of radius $r$ about mass point $\vec{r}_{1}$, each point weighted by the angular harmonic of its direction relative to $\vec{r}_{1}$ (i.e., $e^{i m \theta}$ in two dimensions), and the averages are with respect to all occupied mass points $\vec{r}_{1}$ [8].

The width of the shells used to compute the averages in (3) drops out of the result provided it is small enough, but with finite data sets some compromise must be made to obtain reasonable signal to noise ratio. We typically used shells of $7 \%$ width by radius. In the case of diagonal elements (only), that is, $r=r^{\prime}$ equivalent to $R=1$, shot noise makes a systematically positive contribution to the reading for which we have corrected our data below.

Figure 1 shows contour plots of the shell mass correlations $M_{m}\left(r^{\prime}, r\right)$ as a function of $\ln (r)$ and $\ln \left(r^{\prime}\right)$ for a large site percolation cluster grown at criticality. The structure along the diagonal is direct evidence for the reduction $M_{m}\left(r^{\prime}, r\right)=\mathcal{L}_{m}\left(R=r^{\prime} / r\right)$, and on the basis of such plots we have averaged data parallel to the diagonal, within suitable looking windows, to obtain the curves for $\mathcal{L}_{m}(R)$ shown in Fig. 2. 


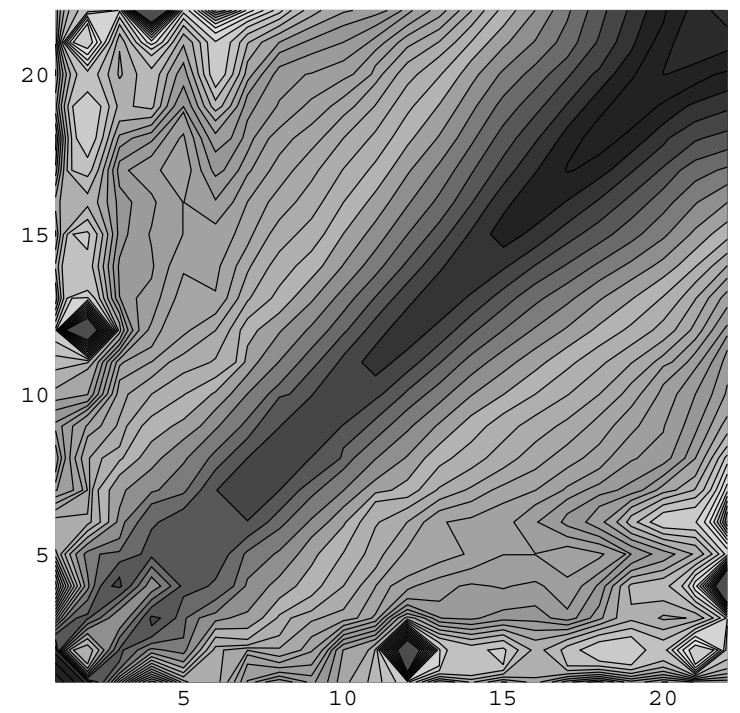

FIG. 1. Contour plot of the cross correlation of local two point function between different length scales, as per Eq. (3) with $m=0$, for a percolation cluster of $1.5 \times 10^{6}$ sites grown on a triangular lattice at the percolation threshold. The axes are labeled proportional to the logarithm of length scale. Contour lines parallel to the diagonal correspond to this reducing to a scale invariant "lacunarity function" depending only on the length scale ratio. Scale invariance breaks down around the edges of the plot where one length scale approaches either the lattice spacing or the sample size.

Figure 3 shows a direct comparison between the corresponding measurements for the outer perimeter set of such a percolation cluster with those for a large self-avoiding walk. The broad agreement (with no adjustable parameters in any of the curves shown) reinforces the claim that these two objects are in the same universality class, as very

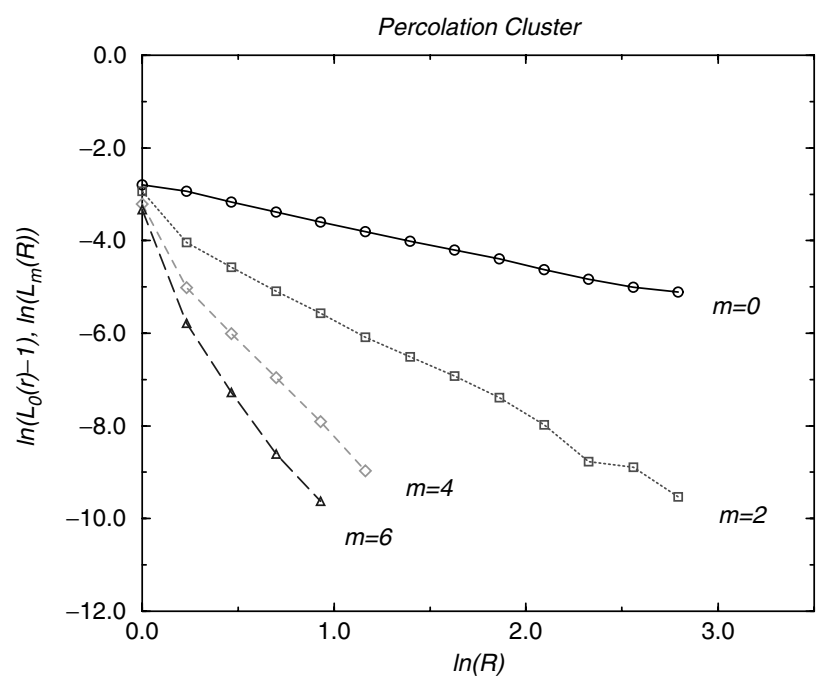

FIG. 2. The different angular harmonics of the lacunarity function for the two-dimensional percolation cluster of Fig. 1. The $m=0$ data correspond to the central part of the contour plot averaged parallel to the diagonal, and the other harmonics are defined analogously in Eq. (3). strongly suggested by Duplantier's calculation [9] of their identical $f(\alpha)$ spectra for the exterior harmonic measure.

II. Connection to the real space renormalization group.-The physical idea behind the renormalization group is that under critical conditions the structure of a system is propagated through a continuous cascade of length scales. This is usually viewed in terms of the propagation of certain (judiciously chosen) renormalized parameters which remain invariant at the fixed point. The linearization of the renormalization group about the fixed point then corresponds to the transmission of perturbations through the length scale cascade.

Our lacunarity function measures the correlation of structure fluctuations between different length scales, and it is natural to suppose that this correlation arises due to propagation. If so, then our measurements of correlation directly probe the linearized renormalization group. More strictly, we need the covariances rather than the correlations to probe propagation, so it is useful to distinguish from lacunarity notation (and rescale) by writing

$$
\mathcal{L} \mathcal{R}_{m}(R)=\frac{\mathcal{L}_{m}(R)-\delta_{m 0}}{\mathcal{L}_{m}(1)-\delta_{m 0}},
$$

where we propose to take the liberty of referring to $\mathcal{L} \mathcal{R}$ as the local renormalization group function.

Our proposal is that if the local two point correlation function structure at radius $r$ about point $\vec{r}_{1}$ is perturbed $C_{2}(r)_{\vec{r}_{1}} \rightarrow C_{2}(r)\left[1+\epsilon_{m}\left(r ; \vec{r}_{1}\right) H_{m}\right]$, where $H_{m}$ is a (normalized) angular harmonic, then the corresponding response at larger length scale $r^{\prime}$ should follow

$$
\epsilon_{m}\left(r^{\prime} ; \vec{r}_{1}\right)=\mathcal{L} \mathcal{R}_{m}\left(r^{\prime} / r\right) \epsilon_{m}\left(r ; \vec{r}_{1}\right) .
$$

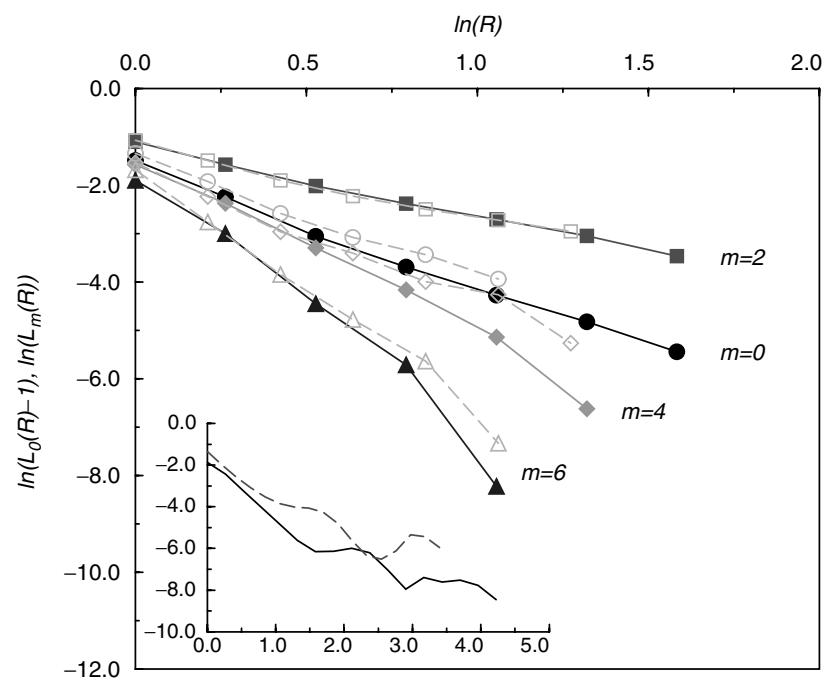

FIG. 3. Angular harmonics of the lacunarity function compared between the outer perimeter of a percolation cluster (as per Fig. 1) and a self-avoiding walk (in two dimensions) of comparable size. The general agreement of these measurements, which contain no adjustable parameters, supports both types of objects being in the same universality class. The inset shows the data for the zeroth harmonic extended to larger length scale ratios. 
The form of this equation is dictated by the requirement that if we apply it to the spontaneous fluctuations of the local two point function, their cross correlation between different length scales is consistent with Eq. (3).

Note that the local renormalization group function defined above is a "shattered" response function, in that it gives the response due to changing the two point correlation at one locality. If we make a global relative perturbation $\epsilon_{m}(r)$ of the local two point correlation at length scale $r$ around every mass point, then the growth of corresponding response defines a "global renormalization group function" $G \mathcal{R}$ through

$$
\epsilon_{m}\left(r^{\prime}\right)=G \mathcal{R}_{m}\left(r^{\prime} / r\right) \epsilon_{m}(r) .
$$

To relate this to the shattered response $\mathcal{L} \mathcal{R}$ we note that the number of (occupied) localities of radius $r$ within a larger vicinity of radius $r^{\prime}=R r$ scales as $R^{D}$ for a simple mass fractal of fractal dimension $D$. Each of these should independently contribute of order $\mathcal{L} \mathcal{R}$ to the global response so we expect the components of $G \mathcal{R}$ to scale as

$$
G \mathcal{R}_{m}(R) \simeq R^{D} \mathcal{L} \mathcal{R}_{m}(R) .
$$

Only even $m$ are of physical consequence here, as the global two point correlation function is by construction an even function restricted to even perturbations.

An important consequence of Eq. (7) is that global perturbations are relevant at large length scales if the corresponding local RG function falls off more slowly than $R^{-D}$ for large $R$.

The case of simple random walks in $d>2$ illustrates and tests these ideas with exact results. The general form of the lacunarity function $\mathcal{L}(R, \theta)$ was first given in Ref. [5], but the angular harmonic decomposition of $\mathcal{L} \mathcal{R}$ gives the much simpler form

$$
\begin{aligned}
\mathcal{L} \mathcal{R}_{0}(R) & =R^{2-d} \\
\mathcal{L} \mathcal{R}_{m}(R) & =\frac{1+R^{2-d}}{2} R^{-m}, \quad m>0 .
\end{aligned}
$$

This gives $G \mathcal{R}_{0}(R) \simeq R^{4-d}, \quad G \mathcal{R}_{2}(R) \simeq R^{0}, \quad$ and $G \mathcal{R}_{4}(R) \simeq R^{-2}$ for large $R$, which all have physical interpretation.

A negative $m=0$ perturbation at length scale $r$ means depressing the incidence of other points at radius $r$ from any given one, corresponding in polymer language to turning on some excluded volume. That the resulting perturbation at larger length scales $r^{\prime}$ grows as $\left(r^{\prime} / r\right)^{4-d}$ corresponds to the well known Fixman perturbation expansion of coil swelling [10].

An $m=2$ perturbation corresponds to the local structure being stretched in some directions and shrunk in others, corresponding for a random walk to anisotropy in the individual walk steps. The scale independence of the resulting response follows from the equivalence of such random walks to affine deformations of isotropic ones, which we discuss as a much more general result below.
An example of an $m=4$ perturbation would be the intrinsic bias of random walks made on a square (more generally hypercubic) lattice, and the decay of the perturbation reflects the known irrelevance of this in the large length scale limit. Analogous interpretations apply to higher orders of lattice bias, for example, $m=6$ and the triangular lattice in two dimensions.

An important issue is whether the propagation of perturbation is necessarily confined to running upwards in length scale. In systems governed by an equilibrium ensemble we can equally expect reverse (inwards) propagation governed by the form of $\mathcal{L} \mathcal{R}$. Thus we are led naturally to predict that for every global perturbation propagating outwards from $r$ to $r^{\prime}$ as $\left(r^{\prime} / r\right)^{-\sigma}$ there is propagation inwards of the same symmetry of perturbation from $r^{\prime}$ to $r$ as $\left(r^{\prime} / r\right)^{-\tau}$ where $\tau=\sigma+D$.

The corresponding inwards perturbations of a random walk can be identified. The zeroth harmonic corresponds to simple confinement (and checks out), while higher harmonics can then be interpreted in terms of the influence of a confining shape. The $m=2$ case, ellipticity, is particularly important because this corresponds to polymer elasticity.

Some of these interpretations can also be checked for less trivial fractals. For the self-avoiding walk (as for the random walk), the nontrivial part of the $m=0$ lacunarity is associated with the walk going from length scale $r$ out to length scale $r^{\prime}$ and coming back again. This is then governed by the probability of an SAW to close a loop, which is known to vary as $N^{-1} \propto r^{\prime-D}$ where $N$ is the number of steps in the walk (to reach radius $r^{\prime}$ ). This leads to $G \mathcal{R}_{0}(R) \simeq R^{0}$ for large $R$, corresponding (correctly) to a change in the excluded volume causing the SAW to dilate uniformly on larger length scales. The inset of Fig. 3 shows our measured data for $L_{0}(R)-1 \propto \mathcal{L} \mathcal{R}_{0}(R)$ out to large $R$ and is consistent with $R^{-D}=R^{-4 / 3}$, albeit the data are noisy. The $m=2$ case for the SAW can be interpreted in terms of the scaling of elasticity for swollen polymers and leads also to $\mathcal{L} \mathcal{R}_{2}(R) \propto R^{-D}$ at large $R$; this checks quite well against the main data in Fig. 3.

We believe a very general result governs the $m=2$ lacunarity due to its RG connection, which explains why in all the fractals considered in this Letter (numerically for the percolation cluster of Fig. 1, and analytically for random and self-avoiding walks),

$$
G \mathcal{R}_{2}(R) \simeq R^{0}
$$

This corresponds to the outwards global $m=2$ perturbation being marginally relevant, and it arises because a $m=2$ perturbation can always be accommodated by a global strain of the metric of space. This should, of course, fail when long-range interactions are important, sensitive to the metric of distance, and therefore Eq. (9) serves as a general test of whether long-range interactions play a role in the underlying physics. 
III. Concluding remarks.-We have presented arguments that the lacunarity function $\mathcal{L}$, or at least its asymptotes, can be viewed as response functions determined by the renormalization group. It is harder to firmly establish just how much of the renormalization group is thereby revealed. Certainly $\mathcal{L}$ is restricted to the linearized renormalization group, because it can probe only fluctuation correlations about the critical-point behavior: in RG terms this means that it can probe only the vicinity of the corresponding fixed point. We have restricted our attention to simple mass fractals, directly characterized in terms of one (positive definite) scalar field, the mass density. We speculate that $\mathcal{L}$ incorporates all the linearized RG relating to how this field might be coupled locally and bilinearly to itself, whereas it cannot know anything about what happens when other fields are introduced.

A rich range of issues remain to be explored. We already mentioned that the odd angular harmonics in the lacunarity function lack a simple response theory interpretation because the two point correlation function must be even. One way to deal with this is to recognize more explicitly the way the lacunarity function depends only on the shape of the triangle of points it correlates, which leads to a parametrization of $\mathcal{L}$ involving only even harmonics. However, it is more interesting to consider structures from irreversible growth where correlating present with future growth is the natural correlation to investigate and odd harmonics are no longer forbidden in such time resolved correlations. We anticipate that problems like the response of diffusion limited aggregation to anisotropy should be analyzed in this way.

In principle our work opens the door to measuring the renormalization group, via lacunarity, directly from real experimental data as opposed to just from computer simulations. We recognize, however, that this may not be easy. Our simulations span some three decades of length scale (i.e., cluster radii of order 1000 units), and even then the match up to expected scaling laws is not always good. The best way forward would appear to be not to target the asymptotic exponents, but rather to compare absolute lacunarity values between experiment, simulation, and theory at moderate $R$. On the theory side, our exact random walk results are extendable to Markov chains, but no other exact results are known (other than exponents).

Finally, while all the fractals discussed in this Letter have close relation to equilibrium critical phenomena, our present definition of lacunarity certainly does not extend trivially to critical phenomena in general. We believe the correct analog of our three point correlation for a mass fractal is a four point correlation of the corresponding critical distributions, the extra point being at infinity. The natural generalization of our lacunarity function to spin models would thus be in terms of a four point correlation function at the critical point, and we look forward to exploring this in future work.

This work was supported by EPSRC, Grant No. GR/L55346, and by the EU under TMR Contracts No. ERBFMBI-CT97-2746 and No. FMRX-CT98-0183.

[1] B. B. Mandelbrot, The Fractal Geometry of Nature (Freeman, San Francisco, 1982).

[2] Y. Gefen, B. B. Mandelbrot, and A. Aharony, Phys. Rev. Lett. 45, 855 (1980).

[3] Y. Gefen, A. Aharony, and B. B. Mandelbrot, J. Phys. A 17, 1277 (1984).

[4] See L.P. Kadaoff, Statistical Physics: Statics, Dynamics and Renormalization (World Scientific, Singapore, 1999), and references therein.

[5] R. Blumenfeld and R.C. Ball, Phys. Rev. E 47, 2298 (1993).

[6] B. B. Mandelbrot, Comptes Rendus (Paris) 278A, 289-292 (1974); 278A, 355-358 (1974).

[7] T. C. Halsey, P. Meakin, and I. Procaccia, Phys. Rev. Lett. 56, 854-857 (1986).

[8] For a fractal represented by $N$ mass points, the direct cost of this operation is order $N^{2}$ unless coarse graining economies are used.

[9] B. Duplantier, Phys. Rev. Lett. 82, 3940-3943 (1999).

[10] M. Fixman, J. Chem. Phys. 23, 1656 (1955). 